\title{
The impact of implementing a Xpert MTB/RIF algorithm on drug-sensitive pulmonary tuberculosis: a retrospective analysis
}

\author{
K. REES ${ }^{1,2}$, N. MUDITAMBI ${ }^{1}$, M. MASWANGANYI ${ }^{3}$, J. RAILTON $^{1}$, \\ J. A. MCINTYRE ${ }^{1,2}$, H. E. STRUTHERS ${ }^{1,4}$, P. B. FOURIE ${ }^{5}$ AND \\ R. P. H. PETERS $S^{1,5,6 *}$ \\ ${ }^{1}$ Anova Health Institute, Johannesburg, South Africa \\ ${ }^{2}$ School of Public Health \& Family Medicine, University of Cape Town, Cape Town, South Africa \\ ${ }^{3}$ Mopani Department of Health, Giyani, South Africa \\ ${ }^{4}$ Division of Infectious Diseases \& HIV Medicine, Department of Medicine, University of Cape Town, Cape \\ Town, South Africa \\ ${ }^{5}$ Department of Medical Microbiology, University of Pretoria, Pretoria, South Africa \\ ${ }^{6}$ Department of Medical Microbiology, School of Public Health and Primary Care (CAPHRI), Maastricht \\ University Medical Centre (MUMC+), Maastricht, The Netherlands
}

Received 22 August 2017; Final revision 18 October 2017; Accepted 6 November 2017;

first published online 6 December 2017

\section{SUMMARY}

Xpert MTB/RIF (Xpert) is the preferred first-line test for all persons with tuberculosis (TB) symptoms in South Africa in line with a diagnostic algorithm. This study evaluates pre- and post-implementation trends in diagnostic practices for drug-sensitive, pulmonary TB in adults in an operational setting, following the introduction of the Xpert-based algorithm. We

retrospectively analysed data from the national TB database for Greater Tzaneen sub-district, Limpopo Province. Trends in a number of cases, diagnosis and outcome and characteristics associated with death are reported. A total of 8407 cases were treated from 2008 until 2015, with annual cases registered decreasing by $31.7 \%$ over that time period (from 1251 to 855 per year). After implementation of Xpert, $69 \cdot 9 \%$ of cases were diagnosed by Xpert, $29 \cdot 4 \%$ clinically, $0 \cdot 6 \%$ by smear microscopy and $0 \cdot 1 \%$ by culture. Cases with a recorded microbiological test increased from $76 \cdot 2 \%$ to $96 \cdot 4 \%$. Cases started on treatment without confirmation, but with a negative microbiological test increased from $7 \cdot 1 \%$ to $25 \cdot 7 \%$. Case fatality decreased from $15 \cdot 0 \%$ to $9 \cdot 8 \%$, remaining consistently higher in empirically treated groups, regardless of HIV status.

Implementation of the algorithm coincided with a reduced number of TB cases treated and improved coverage of microbiological testing; however, a substantial proportion of cases continued to start treatment empirically.

Key words: Diagnostic trend, HIV, tuberculosis, Xpert.

\footnotetext{
* Author for correspondence: R. P. H. Peters, Anova Health Institute, 12 Sherborne Rd, Parktown, Johannesburg 2193, South Africa.

(Email: rph.peters@gmail.com)
}

\section{INTRODUCTION}

The WHO recommends Xpert MTB/RIF (Cepheid, Sunnyvale, CA, USA) as the first-line test for all people suspected of having tuberculosis (TB), where resources allow and particularly for people with HIV-associated or suspected multidrug-resistant TB 
[1]. In 2011, Xpert MTB/RIF (Xpert) was introduced in South Africa, replacing smear microscopy as the initial diagnostic TB test to be performed by national laboratory services. Notably, a revised diagnostic algorithm was implemented in 2014, following simplification of the earlier version which contributed to poor adherence by health care workers, particularly in HIV positive persons with a negative Xpert result [2].

An algorithm based on molecular testing has many potential advantages over one based on smear microscopy, including the need for fewer initial sputum specimens (one instead of two) and fewer patient contacts, increased case-finding due to improved sensitivity and more rapid diagnosis of drug-resistant TB. However, the anticipated increase in TB case-finding has not been evident in trials in South Africa [3] and Brazil [4], possibly due to high background rates of empirical diagnosis and treatment [5]. To date, the national algorithm has not been specifically evaluated but shortcomings have been noted, including poor adherence to the Xpert-negative arm [6].

The impact of the implementation of the Xpertcontaining diagnostic algorithm on the diagnosis of drug-sensitive pulmonary TB in operational settings has not been well described. An enhanced understanding of this impact would benefit the TB programme in South Africa by informing improvements in diagnosis and generate lessons for other TB programmes that may switch to the use of molecular testing. We aimed to evaluate diagnostic trends in patients treated for TB in a rural sub-district of South Africa, in relation to the implementation of Xpert and its accompanying algorithm, using routine data.

\section{METHODS}

\section{Setting}

Greater Tzaneen is the largest sub-district in rural Mopani District, Limpopo Province, South Africa, with most recent population estimates at 390000 . Mopani District is in the second socioeconomic quintile, amongst the poorer districts in South Africa and ranks among the worst districts for years of life lost due to communicable and related diseases [7]. In this sub-district, there are 35 primary care facilities and three district hospitals, all of which manage TB and three public sector laboratories. Xpert testing was first utilised in Greater Tzaneen in 2011.

Prior to the introduction of Xpert testing, two sputum samples taken on consecutive days were sent for smear microscopy (requiring two healthcare worker contacts). If those samples were negative and the person HIV-positive, a third sample was sent for smear microscopy and culture and antibiotic treatment started.

The national algorithm including Xpert testing that is applicable to all people with symptoms of TB was published in 2014 (Supplementary Fig. S1) [8]. The first-line test for all people is one sputum specimen sent for Xpert testing. Following a positive rifampicinsusceptible Xpert test, a sputum specimen should be sent for smear microscopy for monitoring purposes. If the Xpert test is negative, in the case of HIV-positive people, a chest X-ray should be done and a further specimen sent for culture as well as line probe assay or drug sensitivity testing. If the person is HIV negative, antibiotics should be given for 1 week, followed by reassessment.

\section{Study design}

We retrospectively analysed routine data of all the primary healthcare facilities and hospitals in Greater Tzaneen sub-district to understand changes in diagnostic trends within the TB programme. ETR.net is an electronic recording and reporting system used nationally for drug-sensitive TB [9]. Data are captured into paper-based registers at the facility level and submitted to sub-district offices for electronic capturing. Only cases started on TB treatment are recorded in the register.

In this analysis, we included all individuals over 15 years of age started on treatment for drug-sensitive, pulmonary TB in Greater Tzaneen sub-district during the period 2008 to 2015 . We used the database as updated to the end of February 2017, to allow for those starting in 2015 to complete treatment and their outcomes to be captured. To generate population rates, we used district-level mid-year estimates available from Statistics South Africa (http://www.statssa. gov.za/) proportionalised to sub-district level and provincial HIV prevalence figures from the Thembisa model, which synthesises a number of previously developed models of the South African HIV epidemic [10]. Rates are presented per 100000 population.

\section{Definitions}

We assigned TB cases to diagnostic categories based on recorded microbiological tests, assuming that the diagnostic algorithm was followed. Cases were diagnosed 
by Xpert (any case with a positive Xpert result), smear microscopy (any case with a positive smear in the absence of a positive Xpert result), culture (any case with a positive culture in the absence of a positive smear or positive Xpert result), or clinically (any case diagnosed in the absence of a positive microbiological test).

A confirmed TB case was defined as a case started on TB treatment with any recorded positive microbiological test result (the majority of cases started treatment without a TB culture, so a positive culture is not necessary to be considered a confirmed case). An empirically treated TB case was defined as any person starting TB treatment in the absence of a positive microbiological test result [11]. Empirically treated cases were either started on treatment with no microbiological test recorded or with only negative results.

In line with programmatic outcome definitions, any death occurring during the course of TB treatment was classified as a TB death [12]. An early death was defined as occurring in the first 28 days after starting treatment. Treatment success was defined as a case that was either cured (positive baseline smear/culture at start of treatment, negative smear/culture 30 days prior to treatment completion and negative smear/culture during last month of treatment) or completed treatment (completed treatment but did not fulfil required criteria to be assigned as cured).

Ethics approval was obtained from the University of the Witwatersrand Human Research Ethics Committee (Medical), reference M140461.

\section{Data analysis}

Data was exported from ETR.net, collated in Excel and analysed using Stata 13.0 (Stata Corporation, College Station, TX, USA). Duplicate records were excluded, as case information risk being entered into ETR.net multiple times when patients get transferred from one facility to another. Frequencies and proportions are reported to summarise trends in registered cases, demographic characteristics, diagnostic categories and mortality. Rates were calculated by dividing the population by a number of registered TB cases for each year, presented per 100000 . Chi-squared test for trend was used to analyse trends in categorical data. Chi-squared and Mann Whitney tests, as appropriate, were used to compare demographic characteristics within diagnostic categories. Multivariate analysis of factors associated with higher risk of death was conducted using Cox proportional hazards models.

\section{RESULTS}

\section{Study population}

All cases of adults with drug-sensitive pulmonary TB recorded in the ETR.net dataset with treatment start date from 2008 to 2015 in Greater Tzaneen subdistrict were included in this analysis. This means that records of pulmonary TB cases in children $(n=$ 2332) and those with rifampicin mono-resistant or multidrug-resistant TB $(n=58)$ were excluded. Of the 12621 records available, we then excluded individuals that were transferred-in on pulmonary TB treatment $(n=198)$ and those transferred out during TB treatment $(n=3944)$ as initiation respectively outcome data were not available. Finally, de-duplication of records resulted in the removal of another 72 records resulting in a final dataset of 8407 records of adult individuals treated for drug-sensitive TB.

A total of 8407 people over 15 years of age were started on treatment for drug-sensitive pulmonary TB from 2008 to 2015. Of these, 4333 were male $(51 \cdot 5 \%)$ and the median age was 38 years (range 15 110 years). HIV status was recorded for 5717 individuals: 3689 individuals $(64 \cdot 5 \%)$ were HIV-infected, 1701 HIV-negative (29.8\%) and $327(5 \cdot 7 \%)$ had unknown HIV status.

\section{Diagnostic trends}

The number of registered cases decreased over time, from 1251 in 2008 to 855 in $2015(31.7 \%$ decrease, $P<0 \cdot 001)$. Overall, $51 \cdot 5 \%(4333 / 8407)$ of cases were male, with no change over time $(P=0 \cdot 28)$. Median age increased slightly from 37 years in 2008 to 39 years in $2015(P<0 \cdot 001)$. The proportion of cases without a recorded known HIV status decreased dramatically from $93 \cdot 8 \%(1173 / 1251)$ in 2008 to $37 \cdot 4 \%$ (360/962) in 2010 and continued to decrease to $4 \cdot 6 \%(39 / 855)$ in 2015. Of those with a documented known HIV status, HIV-positive cases decreased from $74 \cdot 4 \%(448 / 602)$ in 2010 to $61 \cdot 3 \%(500 / 816)$ in $2015(P<0 \cdot 001)$.

During the time period in question, the antiretroviral therapy (ART) programme in the district expanded from a few thousand people (3395 in 2008) to 62207 on treatment in 2015, (B. Mutasa, Anova Health Institute Mopani District, personal communication) reflecting the expansion of access to ART nationally. This is correlated with a decrease in the number of treated TB cases in the Greater Tzaneen sub-district population; from 1251 cases in 2008 to 855 cases in $2015(P=0 \cdot 01)$. 
In 2010 , the year immediately prior to the introduction of Xpert, diagnosis in Greater Tzaneen was based on smear microscopy $(627 / 962(65 \cdot 2 \%)$ of cases) or on clinical signs and symptoms alone (334/962 (34.7\%) of cases). Culture-based diagnosis occurred in only $0 \cdot 1 \%$ of cases (1/962). By 2015, Xpert almost completely replaced smear microscopy. In this year, the diagnosis was based on Xpert results in $69.9 \%$ of cases (598/ $855)$, clinically in $29 \cdot 4 \%$ of cases $(251 / 855)$ and on smear microscopy in $0.6 \%(5 / 855)$ and culture in $0 \cdot 1 \%(1 / 855)$ of cases (Fig. 1).

In $200830 \cdot 9 \%$ of cases $(387 / 1251)$ were treated empirically, with the proportion progressively increasing over the next 4 years to peak in 2012 at $37.5 \%$ (382/1018), before decreasing to $29 \cdot 4 \%(251 / 855)$ in 2015 (from 2008 to 2015: $P=0 \cdot 11$; from 2011 to 2015: $P<0.001$ ) (Fig. 2). However, cases with a microbiological test outcome (smear microscopy, Xpert or culture) recorded increased from $76 \cdot 2 \%$ $(953 / 1251)$ in 2008 to $96 \cdot 4 \%$ (824/855) in 2015. Cases treated empirically and for whom a negative microbiological test was recorded, increased from $7 \cdot 1 \%$ $(89 / 1251)$ in 2008 to $26 \cdot 2 \%(267 / 1018)$ in 2012 and then stabilised to $25 \cdot 7 \%(220 / 855)$ in 2015 .

Gender was not associated with differences between confirmed and empirically treated cases $(P=0 \cdot 22)$. However, empirically treated cases with documented HIV status were more likely to be HIV positive $(75 \cdot 1 \% ; 1310 / 1745)$ than confirmed cases $(65 \cdot 1 \%$; $2374 / 3645, P<0 \cdot 001)$ and more likely to be older (empirically treated: median age 40 years; confirmed: median age 37 years, $P<0.001$ ).

\section{Population rates}

Estimated rates of treated TB cases dropped by $39 \cdot 7 \%$ between 2008 and 2015, from 480 cases to 290 cases per 100000 people; and 26.6\% between 2011 and 2015, from 395 cases to 290 cases per 100000 people. Rates of confirmed TB dropped by $38.0 \%$ between 2008 and 2015 (from 329 to 204 cases per 100000 people) and unconfirmed TB by $40 \cdot 2 \%$ (from 149 to 85 per 100000 people). The decrease in unconfirmed TB cases was much more pronounced after the introduction of Xpert in 2011 (decrease of $40 \cdot 2 \%$ or $57 \cdot 2$ cases per 100000 population between 2011 and 2015 , compared with $4 \cdot 3 \%$ or $6 \cdot 4$ cases per 100000 population between 2008 and 2011, see Fig. 3).

In HIV positive people, the decrease in overall rates of TB treated between 2011 and 2015 was $37 \cdot 6 \%$, from 2313 to 1442 cases per 100000 HIV positive people.
This was larger for unconfirmed case rates $(47 \cdot 5 \%$, from 862 to 452 cases per 100000 HIV positive people) compared with confirmed case rates $(31.8 \%$, from 1451 to 989 cases per 100000 HIV positive people). However, in the HIV negative population, there was an increase in treatment rates of $3 \cdot 3 \%$, from 118 to 122 cases per $100000 \mathrm{HIV}$ negative people. The rates of treatment of confirmed TB cases in the HIV negative population increased by $17 \cdot 0 \%$ (from 78 to 92 cases per $100000 \mathrm{HIV}$ negative people), while the rate of unconfirmed TB decreased by $23.7 \%$ (from 39 to 30 cases per 100000 HIV negative people) (Fig. 3).

\section{Smear microscopy in Xpert-diagnosed cases}

Of those diagnosed by Xpert, a subsequent smear microscopy test was negative in $6.4 \%$ of cases in 2011 (3/47 cases), increasing to $31 \cdot 7 \%$ of cases in 2015 (181/571 cases) (Table 1).

\section{Outcomes}

Recorded case fatality rates decreased from $14.9 \%$ in 2008 (187 deaths of 1251 TB cases) to $9 \cdot 8 \%$ in 2015 (84 deaths of 855 cases, $P<0 \cdot 001$ ) (Fig. 4). In HIV-positive cases only, these figures were $17.9 \%$ in 2010 (80 deaths of 448 cases) to $12 \cdot 8 \%$ in 2015 (64 deaths of 500 cases, $P<0.001)$ and in HIV-negative, $8 \cdot 4 \%$ in 2010 (13 deaths of 154 cases) to $5 \cdot 1 \%$ in 2015 (16 deaths of 316 cases, $P=0 \cdot 05$ ).

Cases with a successful outcome (cured and completed treatment combined) increased from $71.4 \%$ (893/1251 cases) in 2008 to $84.6 \%$ in 2015 (723/855 cases, $P<0.001)$, largely due to increases in treatment completed. Cases with an unsuccessful outcome (died, defaulted treatment and failed treatment combined) decreased from $24 \cdot 4 \%$ (305/1251 cases) in 2008 to $13 \cdot 5 \%$ in $2015(115 / 855$ cases, $P<0 \cdot 001)$, the majority of these due to deaths.

Case fatality rates were consistently higher in empirically treated cases compared with microbiologically confirmed cases (Fig. 4). Stratifying by HIV status, this remained true in HIV-positive and HIV-negative cases.

In those diagnosed by Xpert, mortality was $8 \cdot 2 \%$ (108/1324) in those with positive subsequent smear microscopy and $6.9 \%(29 / 418)$ in those with negative subsequent smear microscopy results $(P=0 \cdot 42)$.

Multivariate analysis adjusting for year of starting treatment, gender, HIV status and age in years, 


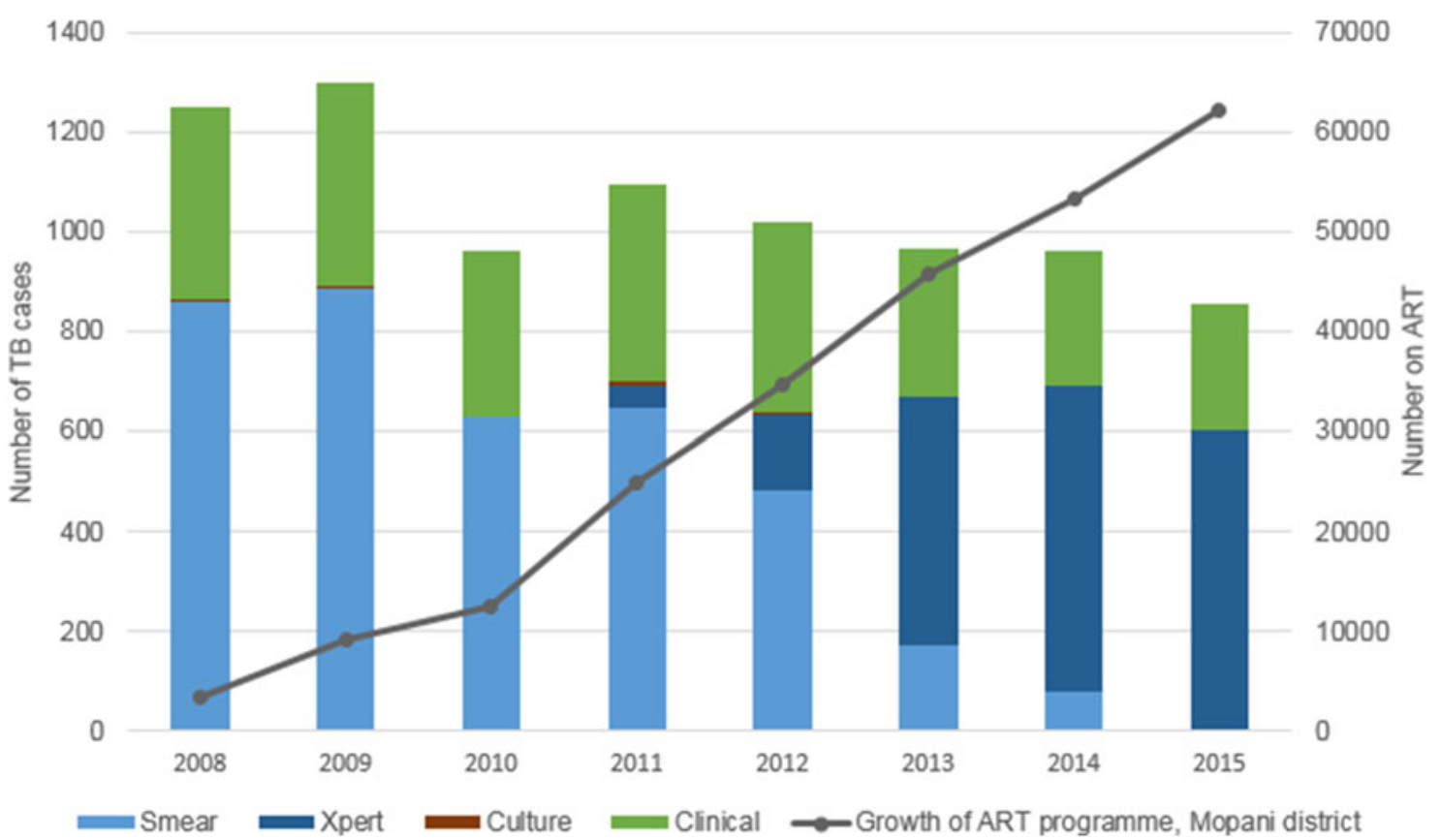

Fig. 1. Method of diagnosis of drug-sensitive, pulmonary TB cases started on treatment in Greater Tzaneen sub-district, 2008-2015. *Growth of ART programme: figures for the whole of Mopani District.

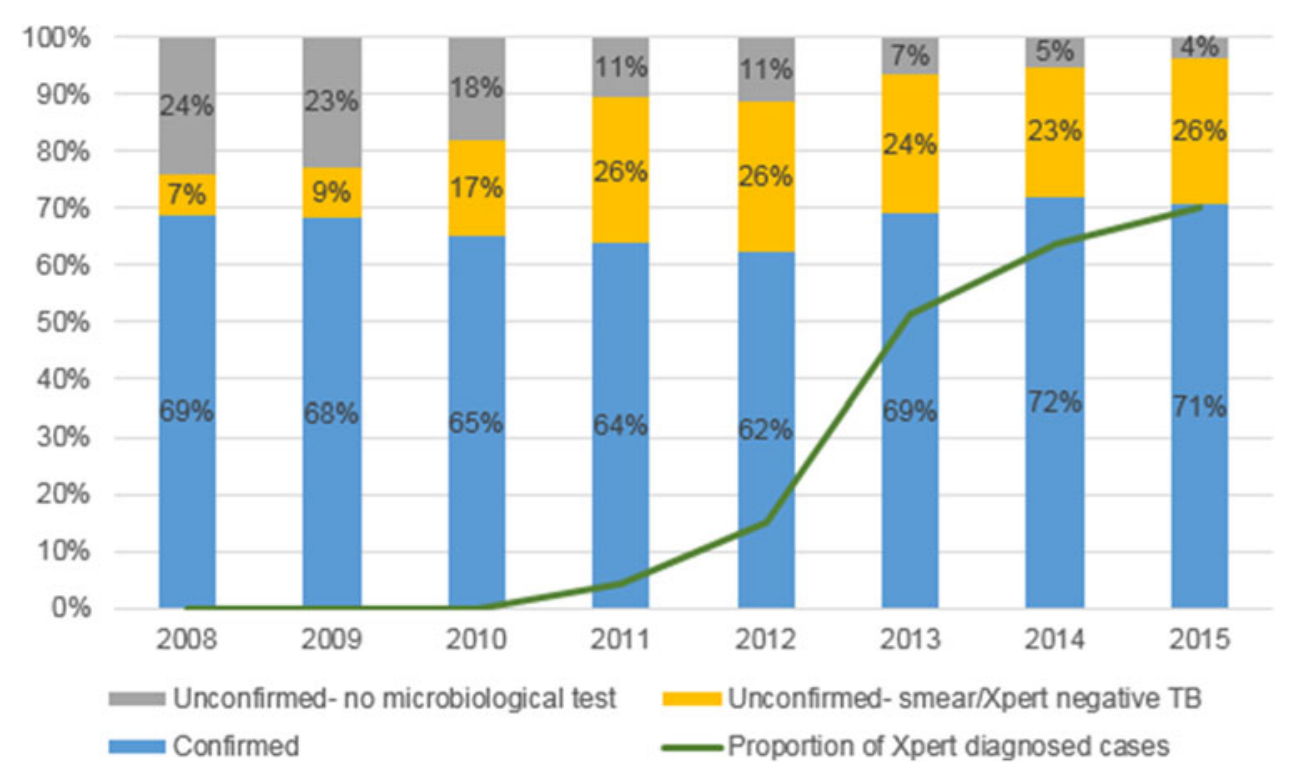

Fig. 2. The proportion of confirmed or empirically treated drug-sensitive, pulmonary TB cases started on treatment in Greater Tzaneen sub-district, 2008-2015.

showed that empirically treated TB was associated with increased risk of death (HR 2.28, 95\% CI 1.97-2.64) (Table 2). This was also true when restricting the analysis to HIV-positive cases only and adjusting for the CD4 count when this data was available, with a hazard ratio of $1.81 \quad(95 \% \mathrm{CI}$ $1 \cdot 43-2 \cdot 30)$.
In those starting treatment in 2014 and 2015, the proportion of early deaths was $40 \cdot 2 \%$ (74 of 184 deaths). There was no significant difference in the proportion of early deaths in HIV-positive compared with HIVnegative cases, men compared with women, or in empirically treated compared with confirmed cases and the median age was similar in those who died early or late. 

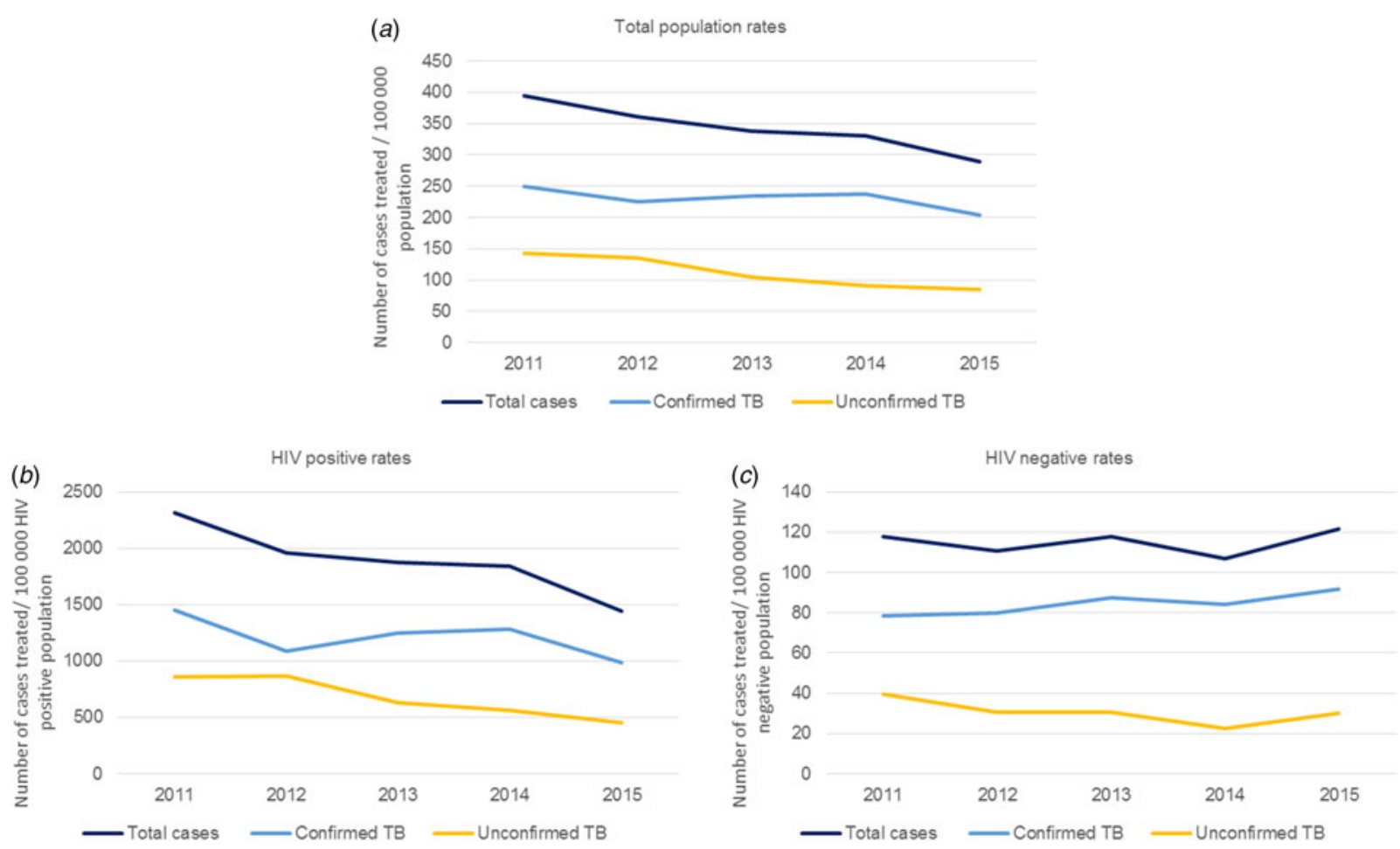

Fig. 3. Population rates (per 100000 people) of drug-sensitive, pulmonary TB cases started on treatment in Greater Tzaneen sub-district, 2011-2015 in (a) the total (b) HIV positive and (c) HIV negative populations.

Table 1. Smear microscopy results of people starting $T B$ treatment based on a Xpert diagnosis of drug-sensitive, pulmonary TB in Greater Tzaneen sub-district, 2008-2015

\begin{tabular}{llllll}
\hline \hline & 2011 & 2012 & 2013 & 2014 & 2015 \\
\hline Xpert diagnosed cases & 47 & 153 & 498 & 611 & 598 \\
Smear positive & 44 & 118 & 352 & 420 & 390 \\
$\quad n(\%)$ & $(93 \cdot 6)$ & $(77 \cdot 1)$ & $(70 \cdot 7)$ & $(68 \cdot 7)$ & $(65 \cdot 2)$ \\
Smear negative & 3 & 19 & 84 & 131 & 181 \\
$\quad n(\%)$ & $(6 \cdot 4)$ & $(12 \cdot 4)$ & $(16 \cdot 9)$ & $(21 \cdot 4)$ & $(30 \cdot 3)$ \\
Smear not done & 0 & 16 & 62 & 60 & 27 \\
$n(\%)$ & $(0 \cdot 0)$ & $(10 \cdot 5)$ & $(12 \cdot 4)$ & $(9 \cdot 8)$ & $(4 \cdot 5)$ \\
\hline \hline
\end{tabular}

\section{DISCUSSION}

We analysed routine programme data for a rural subdistrict in South Africa over the time of Xpert implementation, generating findings that are useful both to our TB programme and to other TB programmes that may be switching from microscopy to molecularbased algorithms in similar settings. The effects of the rapid implementation of Xpert and its accompanying diagnostic algorithm on drug-sensitive, pulmonary $\mathrm{TB}$ in South Africa have not yet been elucidated and analysis of available programme data is a key component of addressing this.

We found that the number of cases registered annually and population rates declined, concurrent with rapid ART programme growth. This is in line with evidence of the decreased risk of TB due to ART in HIV positive people [13, 14], and reports describing concurrent ART scale-up and decreased TB notification in other African countries [15, 16]. In South Africa, the modelled incidence of $\mathrm{TB}$, using both national laboratory service data and national TB database data, peaked in 2008 followed by a decline until 2012 when the study ended and corresponded to increases in ART coverage [17]. Our study showed continuing decreases in treated cases until 2015.

Although rates of treated TB cases declined overall, in the HIV negative population, rates increased due to increases in confirmed TB, while rates of empirically treated TB declined. The decline in rates of empirically treated TB in the HIV negative population despite increased rates overall suggests that the declines in empirically treated TB are not solely due to ART expansion and could be attributed to the routine use of Xpert testing. A population-based study in Cape Town, South Africa similarly found that implementation of Xpert led to decreased rates of empirically 


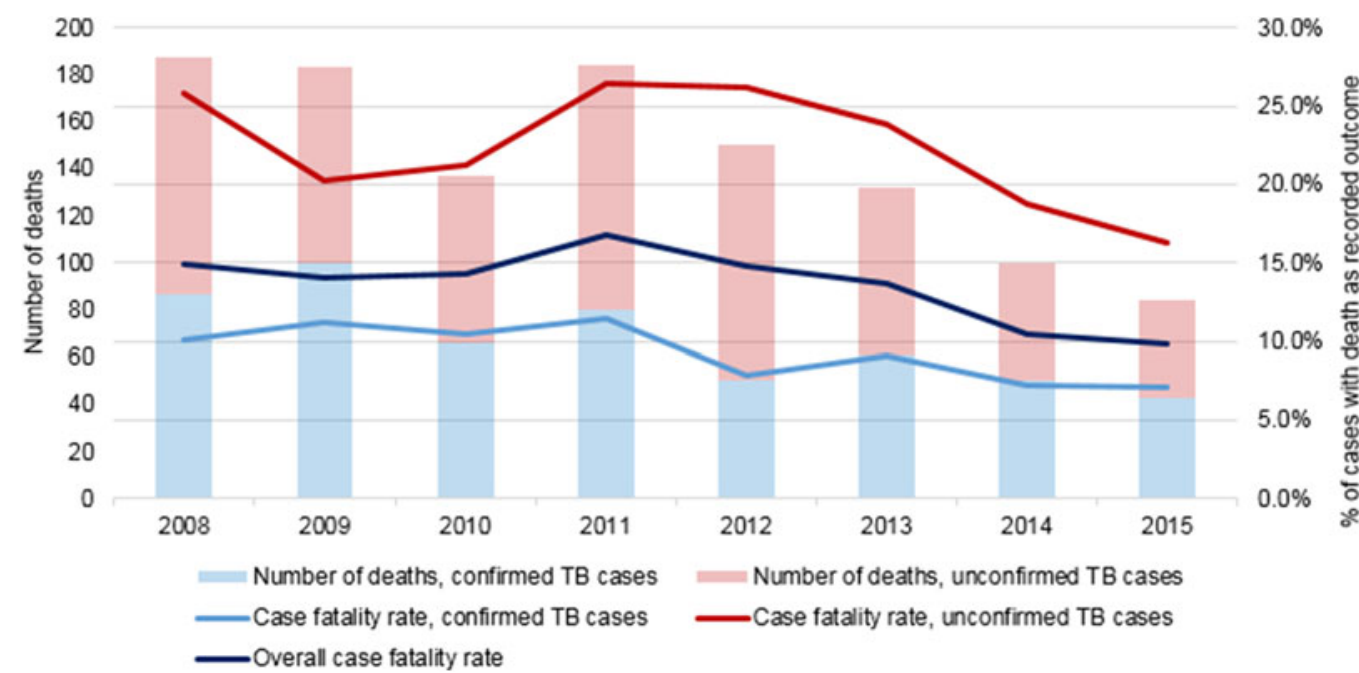

Fig. 4. A number of deaths and case fatality rate in drug-sensitive, pulmonary $\mathrm{TB}$ cases started on treatment, stratified into confirmed TB and unconfirmed TB, in Greater Tzaneen sub-district, 2008-2015.

Table 2. Adjusted Cox proportional hazard models: hazard of death in drug-sensitive, pulmonary TB cases started on treatment in Greater Tzaneen sub-district, 2008-2015

\begin{tabular}{llll}
\hline \hline & Hazard ratio & $95 \%$ CI & \\
\hline All cases & & & \\
Confirmed TB & Reference & & $<\cdot 001$ \\
Empirically treated TB & $2 \cdot 28$ & $1 \cdot 97-2 \cdot 64$ & $<\cdot 001$ \\
Year of starting treatment & $0 \cdot 89$ & $0 \cdot 85-0 \cdot 93$ & $0 \cdot 12$ \\
Female & Reference & & \\
Male & $1 \cdot 10$ & $0 \cdot 95-1 \cdot 28$ & \\
HIV negative & Reference & $1 \cdot 93-2 \cdot 88$ & $<0 \cdot 001$ \\
HIV positive & $2 \cdot 36$ & $1 \cdot 01-1 \cdot 03$ & $<0 \cdot 001$ \\
Age in years & $1 \cdot 02$ & & $<0 \cdot 001$ \\
HIV positive cases only, 2008-2013* & & $1 \cdot 43-2 \cdot 30$ & $<0 \cdot 001$ \\
Confirmed TB & Reference & $0 \cdot 79-0 \cdot 94$ & \\
Empirically treated TB & $1 \cdot 81$ & & \\
Year of starting treatment & $0 \cdot 86$ & $0 \cdot 94-1 \cdot 53$ & $0 \cdot 15$ \\
Female & Reference & $1 \cdot 00-1 \cdot 03$ & $0 \cdot 01$ \\
Male & $1 \cdot 20$ & $0 \cdot 62-0 \cdot 74$ & $<0 \cdot 001$ \\
Age in years & $1 \cdot 01$ & $0 \cdot 67$ & \\
Log CD4 & & & \\
\hline \hline
\end{tabular}

* 2013 is the last year for which CD4 data are available; 1611 individuals had a documented CD4 count with median value of 155 cells $/ \mathrm{mm}^{3}$.

treated TB over time, although overall notification rates did not increase [18]. Besides the introduction of Xpert, there have been other changes in the diagnosis and prevention of TB over this time period, including an increased emphasis on case-finding and the introduction of Isoniazid preventive therapy.

It is possible that overall there would have been an even greater decline in cases without the introduction of the molecular-based diagnostic algorithm. The demonstrated increase in negative smear microscopy tests amongst Xpert-diagnosed cases may reflect the identification of lower bacillary-load infections over time, which would tend to support this supposition. However, these lower bacillary-load infections might have been treated empirically under the microscopybased algorithm. It is also possible that false positives may be contributing to the Xpert-positive, smearnegative group [19]. 
The proportion of people started on TB treatment without a recorded microbiological test decreased substantially, possibly reflecting improvements in adherence to diagnostic guidelines and the improved access to testing that Xpert implementation may have provided. This is a positive sign of improvement in TB programme performance.

The proportion of people started on treatment with only negative microbiological tests increased over time, so that despite increased coverage of microbiological testing, empirically treated TB remained at $28.9 \%$ in 2015. People who were started on treatment empirically experienced higher case fatality rates. This was true of HIV-positive and HIV-negative cases. After adjusting for HIV status, year of starting treatment, age and gender, the hazard of death in empirically treated cases was more than twice that in confirmed cases (HR 2.28, 95\% CI 1.97-2.64). This is a concerning association, that warrants further attention. Potential contributing factors include more advanced immunosuppression (although the association remained in HIV-negative cases and when adjusting for the CD4 count) and the possibility of misdiagnosis or unrecognised comorbid conditions. In addition, clinicians may be more likely to start a patient in poorer overall condition on TB treatment without bacteriological confirmation [5].

Deaths while on TB treatment may be due to a variety of causes, particularly in HIV TB coinfection. Measuring case fatality rates within the TB-programme will not give a complete picture of underlying TB mortality rates in the population, as the deaths of those not started on treatment or lost to follow up are missed and deaths due to other causes are included [20]. The decline in case fatality rates noted in this sub-district is consistent with lower mortality due to TB [21] and all-cause mortality in people on ART, emphasising the impact that increased use of ART has made on reducing mortality among HIV-infected patients with TB [22]. It is important to note that contacts with healthcare workers during TB treatment may represent patients' only contact with the health system, particularly when TB and HIV services are integrated. The way that $\mathrm{TB}$ patients are monitored and healthcare worker awareness of the possibility of misdiagnosis and multiple morbidities are therefore crucial to further decreasing case fatality rates.

Throughout the duration of study, TB cultures were rarely recorded, despite the algorithm's requirement for culture in Xpert-negative HIV positive cases and the high number of these cases started on treatment empirically. Investigating the reasons for the low number of cultures performed in Xpert-negative individuals in our specific setting, including possible issues of access to diagnostic resources and healthcare worker's knowledge of the TB diagnostic algorithm, could be helpful in decreasing empirically treated cases.

This study has illustrated the utility of routine TB data, despite the weaknesses of ETR.net and the results have been used to identify areas of concern within the TB programme, thereby contributing to the strengthening of the programme. Some of the main concerns relating to the database are the lack of a unique identifier (a problem across most South African health information systems) [2], a lack of capacity for data capturing and validation leading to concerns about data quality [2], and no linkage between the TB and HIV databases (Tier.net). However, Anova Health Institute, an organisation with expertise and experience in supporting data quality [23], has been supporting this sub-district for several years including in data quality improvement. Unfortunately, retreatment status was not consistently captured and could not be used in this study. The CD4 count was only captured until 2013 when all people diagnosed with TB were started on ART according to national guidelines, so the use of CD4 count has been limited.

This study has several other limitations. We did not check patient records or paper-based registers for additional information, which could have validated the information obtained and this also meant that we did not have information on chest x-rays, an important element in the diagnosis of Xpert-negative TB [24] and a step in the diagnostic algorithm. Chest $\mathrm{X}$-rays are not universally available at primary care facilities in this rural setting and patients may have to travel at their own expense to obtain one at a higher level of care. Drug-resistant TB is monitored in a separate database and was not included in this study, although identifying rifampicin-resistant TB is one of the key benefits of using Xpert testing. The register used in this study captures data of individuals that are initiated on TB treatment; information of individuals diagnosed with TB but not started on treatment is not included in this analysis and may have resulted in selection. A pre-post design as used in this study has several limitations; although temporality suggests that changes may have occurred in relation to Xpert implementation, other factors could have changed at the same time and contributed to the observed 
association, e.g. changes in ART coverage and the clinical management of TB [25]. Finally, our study setting is a rural sub-district in a resource-constraint area of South Africa; care should be applied when extrapolating our results to other settings.

However, the information obtained from this analysis adds to our current understanding of the TB programme in South Africa and highlights areas that need more attention. The high rates of empirically-treated $\mathrm{TB}$, even with access to Xpert testing, are in line with other evidence from this setting [26, 27], and remained high despite improvements in coverage of microbiological testing. Our data support prior calls to address the weaknesses in the current algorithm in terms of diagnosing TB in HIV positive people with Xpert-negative results and the lack of guidance in monitoring these cases [27, 28]. Repeating Xpert testing rather than culture following an initial negative Xpert has been suggested and found to be potentially cost saving in the context of the South African TB programme [29]. Poor adherence to the algorithm noted in other South African settings [6], and the poor use of TB culture in this study, supports this idea, although more comprehensive guidelines for diagnosis and follow up are also required.

\section{SUPPLEMENTARY MATERIAL}

To view supplementary material for this article, please visit https://doi.org/10.1017/S0950268817002746

\section{ACKNOWLEDGEMENTS}

We thank the staff of the Mopani District Department of Health for their contributions to this study. Anova Health Institute is supported by the US President's Emergency Plan for AIDS Relief (PEPFAR) program via the US Agency for International Development (USAID) under Cooperative Agreement No. AID674-A-12-00015. The views expressed in this manuscript do not necessarily reflect those of PEPFAR or USAID.

\section{DECLARATION OF INTEREST}

None.

\section{ETHICAL STANDARDS}

The authors assert that all procedures contributing to this work comply with the ethical standards of the relevant national and institutional committees on human experimentation and with the Helsinki Declaration of 1975, as revised in 2008.

\section{REFERENCES}

1. World Health Organisation. Automated Real-Time Nucleic Acid Amplification Technology for Rapid and Simultaneous Detection of Tuberculosis and Rifampicin Resistance: Xpert MTB/RIF Assay for the Diagnosis of Pulmonary and Extrapulmonary $T B$ in Adults and Children. Policy update. Geneva: World Health Organisation, 2013.

2. Churchyard GJ, et al. Tuberculosis control in South Africa: successes, challenges and recommendations. South African Medical Journal 2014; 104(3): 244.

3. Naidoo P, et al. Comparing tuberculosis diagnostic yield in smear/culture and Xpert(R) MTB/RIF-based algorithms using a non-randomised stepped-wedge design. PLOS ONE 2016; 11(3): e0150487.

4. Durovni B, et al. Impact of replacing smear microscopy with Xpert MTB/RIF for diagnosing tuberculosis in Brazil: a stepped-wedge cluster-randomized trial. PLOS Medicine 2014; 11(12): e1001766.

5. Theron G, et al. Do high rates of empirical treatment undermine the potential effect of new diagnostic tests for tuberculosis in high-burden settings? The Lancet Infectious Diseases 2014; 14(6): 527-532.

6. McCarthy K, et al. What happens after a negative test for tuberculosis? Evaluating adherence to TB diagnostic algorithms in South African primary health clinics. Journal of Acquired Immune Deficiency Syndromes 2016; 71(5): e119-e126.

7. Massyn N, et al. District Health Barometer 2015/16. Durban: Health Systems Trust, 2016.

8. Republic of South Africa Department of Health. National Tuberculosis Management Guidelines 2014. Pretoria: Fishwicks PTA, 2014.

9. Nadol P, et al. Electronic tuberculosis surveillance systems: a tool for managing today's TB programs. International Journal of Tuberculosis and Lung Disease 2008; 12(3): S8-S16.

10. Johnson LF, Dorrington RE, Moolla H. Modelling the Impact of HIV in South Africa's Provinces. Centre for Infectious Disease Epidemiology and Research working paper. Cape Town: University of Cape Town, 2016.

11. Churchyard GJ, et al. Xpert MTB/RIF versus sputum microscopy as the initial diagnostic test for tuberculosis: a cluster-randomised trial embedded in South African roll-out of Xpert MTB/RIF. The Lancet Global Health 2015; 3(8): e450-e457.

12. World Health Organisation. Definitions and Reporting Framework for Tuberculosis - 2013 Revision. Geneva: World Health Organisation, 2013.

13. Badri M, Wilson D, Wood R. Effect of highly active antiretroviral therapy on incidence of tuberculosis in South Africa: a cohort study. The Lancet 2002; 359(9323): 2059-2064. 
14. Nicholas S, et al. Incidence of tuberculosis in HIV-infected patients before and after starting combined antiretroviral therapy in 8 Sub-Saharan African HIV programs. Journal of Acquired Immune Deficiency Syndromes 2011; 57: 311-318.

15. Kanyerere H, et al. Scale-up of ART in Malawi has reduced case notification rates in HIV-positive and HIV-negative tuberculosis. Public Health in Action 2016; 6(4): 247-251.

16. Takarinda KC, et al. Declining tuberculosis case notification rates with the scale-up of antiretroviral therapy in Zimbabwe. Public Health in Action 2016; 6(3): 164-168.

17. Nanoo A, et al. Nationwide and regional incidence of microbiologically confirmed pulmonary tuberculosis in South Africa, 2004-12: a time series analysis. The Lancet Infectious Diseases 2015; 15(9): 1066-1076.

18. Hermans $\mathbf{S}$, et al. The impact of the roll-out of rapid molecular diagnostic testing for tuberculosis on empirical treatment in Cape Town, South Africa. Bulletin of the World Health Organization 2017; 95(8): 554-563.

19. Theron G, et al. Xpert MTB/RIF results in patients with previous tuberculosis: can we distinguish true from false positive results? Clinical Infectious Diseases 2016; 62(8): 995-1001.

20. Korenromp E, et al. The measurement and estimation of tuberculosis mortality. International Journal of Tuberculosis and Lung Disease 2009; 13(3): 283-303.

21. Nglazi MD, et al. The impact of HIV status and antiretroviral treatment on TB treatment outcomes of new tuberculosis patients attending co-located $\mathrm{TB}$ and ART services in South Africa: a retrospective cohort study. BMC Infectious Diseases 2015; 15: 536.

22. Slaymaker E, et al. How have ART treatment programmes changed the patterns of excess mortality in people living with HIV? Estimates from four countries in East and Southern Africa. Global Health Action 2014; 7: 22789.

23. Myburgh $\mathbf{H}$, et al. Implementation of an electronic monitoring and evaluation system for the antiretroviral treatment programme in the Cape Winelands district, South Africa: a qualitative evaluation. PLOS ONE 2015; 10(5): e0127223.

24. Theron G, et al. Do adjunct tuberculosis tests, when combined with Xpert MTB/RIF, improve accuracy and the cost of diagnosis in a resource-poor setting? European Respiratory Journal 2012; 40(1): 161-168.

25. Theron G, et al. Feasibility, accuracy, and clinical effect of point-of-care Xpert MTB/RIF testing for tuberculosis in primary-care settings in Africa: a multicentre, randomised, controlled trial. Lancet 2014; 383: 424 435 .

26. Budgell EP, et al. Outcomes of treatment of drugsusceptible tuberculosis at public sector primary healthcare clinics in Johannesburg, South Africa: a retrospective cohort study. South African Medical Journal 2016; 106(10): 1002-1009.

27. Hanrahan CF, et al. Time to treatment and patient outcomes among TB suspects screened by a single point-of-care Xpert MTB/RIF at a primary care clinic in Johannesburg, South Africa. PLOS ONE 2013; 8(6): e65421.

28. Peter J, Theron G. The progression of TB diagnosis in the HIV era: from microscopes to molecules and back to the bedside. Continuing Medical Education 2011; 29(10): 404-408.

29. Schnippel K, et al. Diagnosing Xpert MTB/RIF negative TB: impact and cost of alternative algorithms for South Africa. South African Medical Journal 2013; 103(2): 101-106. 